\title{
Self-consistent method for optimization of parameters of diode temperature sensors
}

\author{
N. R. Kulish, Yu. M. Shwarts, V. L. Borblik, Ye. F. Venger, V. N. Sokolov \\ Institute of Semiconductor Physics of NASU, 45, Prospect Nauki, 252028 Kiev, Ukraine.
}

\begin{abstract}
In the framework of the diffusion transport model through an abrupt asymmetric $p n$-junction, the ideality factor of which is assumed to be equal to unity, and with the help of criteria commonly used to describe theoretically the semiconductor diode structures, the relations are obtained for estimation of parameters of the diode temperature sensor. The set of these parameters provides either the maximum extent of a thermometric characteristic toward the higher temperature range, or maximum sensitivity of the diode temperature sensor. For $\mathrm{Ge}, \mathrm{Si}, \mathrm{GaAs}$ diode temperature sensors with $n^{+} p$ - and $p^{+} n$ - junctions the limits of thermometric characteristics were determined, together with temperature dependencies of sensitivity, static and dynamic resistance calculated for cases of the maximum length of the thermometric characteristic and of maximum sensitivity. It has been shown that experimentally measured characteristics of diode temperature sensors are within the ranges determined by the limiting characteristics. The ways of further improvement of diode temperature sensors are discussed.
\end{abstract}

Keywords: temperature, sensor, $p^{+} n$ - junction, ideality factor, thermometric characteristic, sensitivity.

Paper received 01.12.98; revised manuscript received 03.06.99; accepted for publication 12.07.99.

\section{Introduction}

The following main properties of diode temperature sensors (DTS) are critical for a consumer: 1) range of measured temperatures; 2) accuracy of temperature measurement; 3 ) sensitivity; 4) magnitude of supply current. The values of specific characteristics as well as the interrelation between them are determined by the set of electrophysical parameters of a semiconductor (bandgap width, emitter and base doping levels, concentration of deep centers) and by design parameters of the diode $\left(n^{+} p\right.$ - or $p^{+} n$-junction, $p n$ - junction area and depth, base length). Previously the effects of only some of mentioned factors on the DTS characteristics were studied, namely: of the base doping level on sensitivity $[1,2]$; of the supply current on sensitivity and the range of measured temperatures [2-4]; of the junction type $\left(n^{+} p\right.$ or $\left.p^{+} n\right)$ on the thermometric characteristic (TMC) [2]. In these papers neither an optimization of main DTS characteristics was done, nor a question about the possibility of such an optimization was raised.

The aim of this paper is to develop the self-consistent procedure for determination of electrophysical and design parameters of DTS based on the given values of the range of measured temperatures, the temperature measurement accuracy and sensitivity. The paper consists of three section. In the first section the general equations are given establishing relations between diode electrophysical and design parameters and DTS characteristics. It is shown that two sets of parameter values can be distinguished, the first one providing a maximally wide range of measured temperatures, and the second one giving the maximum DTS sensitivity. In the second section the self-consistent procedure of determination of these parameters for $\mathrm{Ge}, \mathrm{Si}$ and $\mathrm{GaAs}$ DTS was carried out. In the third section the calculated temperature dependencies of the voltage drop across $n^{+} p$ - and $p^{+} n$-junctions, sensitivity, static and dynamic resistance of Ge-, Si- and GaAs-based DTS are presented, corresponding either to the maximum length of TMC or to maximum sensitivity of DTS. The comparison has been made of calculated and experimental DTS characteristics. In conclusion, the ways of an improvement of diode temperature sensors are briefly discussed. 


\section{N. R. Kulish et al.: Self-consistent method for optimization of parameters ...}

\section{Relations connecting the electrophysical and design parameters of the diode with characteristics of the diode temperature sensor}

In conducting the relations which establish the connection between electrophysical and design characteristics of the diode and characteristics of DTS the following criteria were used: 1) the maximum value of temperature registered by DTS is assumed to be below the temperature corresponding to the transition to intrinsic conduction of the base and is determined by the temperature when the voltage at $p n$-junction is equal to the thermal voltage; 2 ) the base doping level has to be less than the value at which the effect of tunnel processes on transport through $p n$-junction should be taken into account; 3 ) the signal-to-noise ratio should be greater than unity.

The resulting relations will, naturally, depend on the specific transport mechanism through $p n$-junction, i.e. on the form of the temperature dependence of the current and on the magnitude of the voltage drop across $p n$-junction. In this paper the procedure of determination of the relation linking the electrophysical and design parameters with DTS characteristics is carried out using the model of the diffusion transport mechanism for an abrupt asymmetric junction. During this procedure 1) the one-dimensional model of pn-junction is used with heavily doped (in assumption of fully ionized impurities and non-degenerated carriers) and uniform $p$ - and $n$-regions, the voltage drops at which are negligibly low; 2) the voltage drop at Ohmic contacts is neglected; 3 ) the width of the space charge region of pnjunction is supposed to be significantly less than the majority carriers diffusion length; 4) the injection level is supposed to be low.

Under these conditions the dependence of the current density $J$ on the voltage drop $U$ across $p n$-junction has the form [5]

$$
J=J_{s}[\exp (q U / k T)-1],
$$

where $J_{s}$ is the diffusion saturation current density $[6,7]$

$$
J_{s}=\left(q D_{b} / L_{b}\right)\left[N_{b} t h\left(d / L_{b}\right]^{-1} n_{i}^{2},\right.
$$

$n_{i}=\left(N_{c} N_{v}\right)^{1 / 2} \exp \left(-E_{g} / 2 k T\right)$ - is the intrinsic carrier concentration, $N_{c}$ and $N_{v}$ are effective densities of states in the conduction and valence band, respectively, $q$ is the electron charge, $k$ is the Boltsmann constant, $T$ is temperature, $L_{b}=\left(D_{b} \tau_{b}\right)^{1 / 2}$ and $D_{b}=\mu_{b} k T / q$ are the diffusion length and coefficient of diffusion of minority carriers, $N_{b}, \mu_{b}$ and $\tau_{b}$ are impurity concentration, mobility and minority carrier lifetime in the base, respectively, $d$ is the base length, $E_{g}$ is the width of the semiconductor bandgap, the temperature dependence of which is determined by the equation [8]

$$
E_{g}(T)=E_{g}(0)-a T^{2} /(T+b),
$$

where $E_{g}(0)$ is the bandgap width at absolute zero temperature, $a$ and $b$ are constants. In the case of $p^{+} n$-junction, a)

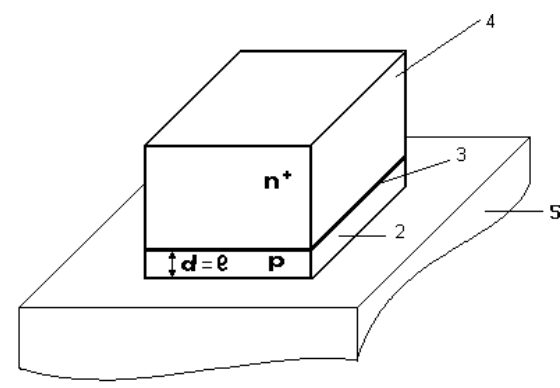

b)

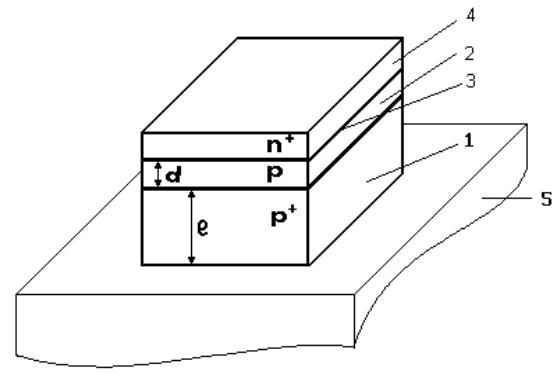

c)

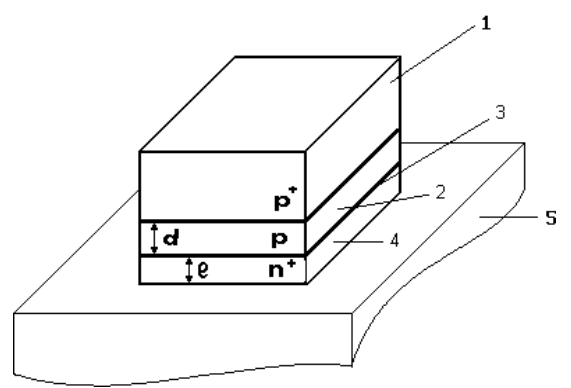

Fig. 1. Simplified schemes of variants of $p n$-junction location in respect to the base of the package: a) ideal, b) typical, c) preferrable. 1 - heavily doped substrate, 2 - base, 3 - pn-junction, 4 - emitter, 5 - base of the package.

$N_{b}=N_{D}$, where $N_{D}$ is the donor concentration, $\tau_{b}=\tau_{p}, \mu_{b}=\mu_{p}$, $D_{b}=D_{p}, L_{b}=L_{p}$, and, in the case of $n^{+} p$-junction, $N_{b}=N_{A}$, where $N_{A}$ is the acceptor concentration, $\tau_{b}=\tau_{n}, \mu_{b}=\mu_{n}, D_{b}=D_{n}$, $L_{b}=L_{n}$. Here the subscript $n$ denotes the parameters related to electrons, and subscript $\mathrm{p}$ is related to holes.

At a given value of the current density $J$, from (1) the equation of the thermometric characteristic of the diode temperature sensor follows

$$
U(T)=(k T / q) \ln \left[\left(J / J_{s}\right)+1\right]
$$

The intrinsic carrier concentration, and, the saturation current density increase with increasing temperature. At some temperature $T=T_{m}$ the saturation current density $J_{s}$ approaches to the operation current density $J$. In this case the voltage drop $U$ across the $p n$-junction becomes of the order $k T / q$. Temperature $T_{m}$, corresponding to this condition, is the maximum (limiting) temperature which can be measured with the diode thermometer from the voltage drop across the $p n$-junction.

Assuming $q U=k T_{m}$ in (4) and considering, for definiteness, $n^{+} p$-junction, we obtain the transcendent equation for determination of the limiting temperature 


\section{N. R. Kulish et al.: Self-consistent method for optimization of parameters ...}

$$
T_{m}=\frac{E_{g}\left(T_{m}\right) / k}{\ln \left\{\frac{4(e-1)\left(m_{e} m_{h}\right)^{3 / 2}\left(2 \pi k T_{m} / h^{2}\right)^{3}\left[q \mu_{n}\left(T_{m}\right) k T_{m}\right]^{1 / 2}}{J N_{A}\left[\tau_{n}\left(T_{m}\right)\right]^{1 / 2} t h\left\{d /\left[\mu_{n}\left(T_{m}\right) k T_{m} \tau_{n}\left(T_{m}\right) / q\right]^{1 / 2}\right\}}\right\}}
$$

where $m_{e}$ и $m_{h}$ are the effective masses of density of states in conduction and valence bands, respectively.

It can be seen from (5) that $T_{m}$ is shifted towards the higher temperatures for semiconductors with the greater bandgap width $E_{g}$, and, for a specific semiconductor, at higher operation current density and doping level. In the short-base diodes $\left(d<<L_{n}\right)$ temperature $T_{m}$ does not depend on $\tau_{n}$ and is lower for shorter base lengths $d$, and in the long-base diodes $\left(d>>L_{n}\right)$ it is independent of $d$ and is higher for greater $\tau_{n}$. While calculating $T_{m}$ one should take into account that $E_{g}, \mu_{n}, \tau_{n}$ are the temperature-dependent parameters, and $N_{A}, J$ and $d$ do not depend on $T$. The analysis of the influence of semiconductor parameters on the magnitude of $T_{m}$ we will begin by the study of the effect of doping impurity concentration and of the current density.

Concentrations of donors $N_{D}$ and acceptors $N_{A}$. The emitter impurity concentration $N_{D}$ has an upper limit determined by the solubility limit, and that of the base impurity concentration $N_{A}$ is determined either by an avalanche breakdown of $p n$-junction, or by electron bandto-band tunneling. Here the diode without band-to-band tunneling is considered, therefore the upper limit of the impurity concentration in the base $N_{A m}$ is estimated from the approximate relation between the avalanche breakdown voltage $U_{b}$ and $N_{A m}$ [9]

$$
U_{b} \approx 60\left[E_{g}(300 K) / 1.1\right]^{3 / 2}\left(N_{A m} / 10^{16}\right)^{-3 / 4} \text {, }
$$

where $E_{g}(300 \mathrm{~K})$ is the bandgap width at $300 \mathrm{~K}$. If the values of $E_{g}(300 \mathrm{~K})$ in $\mathrm{eV}$ and of $N_{A m}$ in $\mathrm{cm}^{-3}$ are inserted into (6), the value $U_{b}$ in volts can be obtained. It follows from (6) that the base doping level can be increased only if the avalanche breakdown voltage $U_{b}$ is reduced.

Operation current density. When the current is flowing through $p n$-junction, the Joule heating takes place. As a result, the temperature of pn-junction is higher by $\Delta T_{H}$ than the temperature of the semiconductor substrate being in contact with ambient. In this case $\Delta T_{H}$ is connected with the thermal power density $P / S$ by the relation $[10,11]$

$$
\Delta T_{H}=P l / S \lambda,
$$

where $P=I U(T), I=J S$ is the operation current, $\lambda(T)$ is the coefficient of thermal conductivity, $S$ is the $p n$-junction area (Fig. 1), from which the thermal diffusion toward the sample surface occurs, $l$ is the $p n$-junction depth in respect to the outer surface of the chip. Actually, $\Delta T_{H}$ is the systematic measuring error due to Joule heating of $p n$-junction.

From (7) the expression for the upper limit of the operation current density it follows that

$$
J_{m}=\Delta T_{H} \lambda(T) / U(T) l .
$$

To find $J_{m}$ the maximum values of $\mathrm{U}(\mathrm{T})$ and $l$ and minimum values of $\lambda(T)$ and $\Delta T_{H}$ should be inserted into (8). Then the systematic error due to overheating will not exceed a given value $\Delta T_{H}$ for the whole range of the thermometric characteristic.

Upper limit of temperature. According to (5) the temperature $T_{m}$ can be varied in a wide range depending on the specific set of semiconductor parameters. In the considered transport model the upper limit of measured temperatures should be less than the temperature $T_{i}$ of transition to intrinsic conduction in the diode base [12]. We will assume that, in the framework of the given model, the greatest value of $T_{m}$ is equal to the temperature $T_{i}^{\prime}$ at which the intrinsic charge carrier concentration is as small as $10 \%$ of the base acceptor concentration $N_{A}$. This limiting value of temperature $T_{m}=T_{i}^{\prime}$ will then be determined from the transcendent equation

$\left(0.1 N_{A}\right)^{2}=N_{c}\left(T_{i}^{\prime}\right) N_{\mathrm{v}}\left(T_{i}^{\prime}\right) \exp \left[-E_{g}\left(T_{i}^{\prime}\right) / k T_{i}^{\prime}\right]$.

If the values $T_{m}=T_{i}^{\prime}, J=J_{m}, N_{A}=N_{A m}$ from (9), (8), (6) are inserted into (5), then the equation (5) will define the relation between other parameters: $d, l, \tau_{n}$.

Sensitivity. According to (4) we find the sensitivity $\alpha(T)=d U(T) / d T$ :

$$
\begin{aligned}
& \alpha(T)=\frac{U(T)}{T}-\frac{k J}{q\left(J+J_{s}\right)}\left[\frac{7}{2}+\frac{2 d / L_{n}}{s h\left(2 d / L_{n}\right)} \frac{d\left(\ln L_{n}\right)}{d(\ln T)}+\right. \\
& \left.+\frac{1}{2} \frac{d(\ln \mu)}{d(\ln T)}-\frac{1}{2} \frac{d\left(\ln \tau_{n}\right)}{d(\ln T)}+\frac{E_{g}}{k T}-\frac{1}{k} \frac{d E_{g}}{d T}\right] .
\end{aligned}
$$

For determination of parameters most affecting the magnitude of $\alpha(T)$ far from the point $T=T_{m}$, we simplify (10) taking into account, that until $q U>>k T$, the relation $J>>J_{s}$ is valid. The estimations show that the term $E_{g} / k T$ dominates in the brackets of (10). In these approximations the equation (10) is reduced to the form

$$
\alpha(T)=-\frac{k}{q} \ln \left\{\frac{4\left(q \mu_{n} k T\right)^{1 / 2}\left(2 \pi k T / h^{2}\right)^{3}\left(m_{e} m_{h}\right)^{3 / 2}}{J N_{A} \tau_{n}^{1 / 2} \operatorname{th}\left(d / L_{n}\right)}\right\} .
$$

It follows from (11) that $\alpha(T)$ does not depend on the semiconductor bandgap width. Since $|\alpha| \sim 1 / \ln \left(J N_{A}\right)$ an increase of sensitivity should be observed at the lower current density and doping impurity concentration in the base, or, in other words, with decreasing the limiting temperature $T_{m}$. In the short-base diodes the sensitivity (11) does not depend on the minority carrier lifetime $\tau_{n}$ and increases, in absolute magnitude, with reduction of the base length $d$, and in the long-base diodes it increases with reduction of $\tau_{n}$ and does not depend on the base length.

To find $\alpha(T)$ at $T=T_{m}$ the voltage $U\left(T_{m}\right)$ in (10) is substituted by its value $k T_{m} / q$, and $J$ is determined from the fol- 


\section{N. R. Kulish et al.: Self-consistent method for optimization of parameters ...}

lowing relation

$J=(e-1) J_{s}\left(T_{m}\right)$,

which follows from (4). As a result we get the approximate expression

$\alpha\left(T_{m}\right)=-\frac{e-1}{e} \frac{E_{g}\left(T_{m}\right)}{q T_{m}}$.

According to (13) at the limiting point of TMC $T=T_{m}$ the sensitivity is fully determined by properties of material which is used in a technical realization of DST.

Operation current and $\boldsymbol{p n}$-junction area. According to $[8,13]$ thermal and shot noise dominate in the diode temperature sensors. In the considered transport model the mean-square value of the noise current $\left\langle i_{N}^{2}\right\rangle$ is described by the expression $[8,14]$

$<i_{N}^{2}>=(4 k T G-2 q I) B_{\omega}$,

where $G=d I / d U=q\left(I+I_{S}\right) / k T$ is the differential conductance, $B_{\omega}=1 / 2 \pi \tau$ is the bandwidth, $\tau$ is the minority carrier lifetime, $\omega$ is the circular frequency. In the right-hand side of (14) the expression in parentheses determines the spectral density of current fluctuations $S_{i}(\omega)$ for low frequencies and does not depend on the fluctuation frequency $\omega$. In a small vicinity of some point of the current-voltage characteristic with the dynamic resistance $r=d U / d I$ the spectral density of current fluctuations $S_{i}(\omega)$ is connected with the spectral density of voltage fluctuations $S_{U}(\omega)$ by the relation $S_{U}(\omega)$ $=r^{2} S_{i}(\omega)$. Then the mean-square value of the noise voltage $<U_{N}^{2}>$ can be written as

$<U_{N}^{2}>=\frac{(k T)^{2}}{\pi q \tau_{n}} \frac{I+2 I_{s}}{\left(I+I_{s}\right)^{2}}$.

To find the systematic error $\Delta T_{N}$ due to the $p n$-junction noise we use the relation

$|\alpha(T)| \Delta T_{N}=U_{N}$,

where $U_{N} \equiv \sqrt{\left\langle U_{N}^{2}\right\rangle}$. Inserting the expression (15) for $<U_{N}^{2}>$ into (16) we come to the relation which permit estimation of the minimum operation current value resulting in a given error $\Delta T_{N}$

$I_{\min }=\frac{1}{2} I_{0}\left(1-2 \frac{I_{s}}{I_{0}}+\sqrt{1+4 \frac{I_{s}}{I_{0}}}\right)$

where

$I_{0} \equiv \frac{(k T)^{2}}{\pi q \tau_{n}\left(\alpha \Delta T_{N}\right)^{2}}$.

For currents $I>I_{\min }$ the systematic error of the temperature measurement related to the noise will be less than $\Delta T_{N}$. At $4 I_{s} / I_{0}<<1$ it follows from (17) that $I_{\min }=I_{0}$.
The minimum value of the area $S_{\min }$ of $p n$-junction can be easily found from the known values of the current density $J_{m}(8)$ and current $I_{\min }(17)$

$S_{\min }=I_{\min } / J_{m}$.

The maximum values of the pn-junction area $S_{\max }$ and of the current $I_{\max }$ we will determine from the following technological restrictions. Manufacture of semiconductor devices has the tendency to minimization of the device size which is provided by its fabrication technology. The minimum size of a chip is determined by the technique of wafer sawing and is $500 \times 500 \times 400 \mu \mathrm{m}^{3}[15]$. With account of sides of the crystal deteriorated by sawing, the $p n$-junction area reduces to the values $S_{\max }=300 \times 300 \mu \mathrm{m}^{2}$. Then the maximum value of operation current is

$I_{\max }=S_{\max } J_{m}$.

Resistance of thermodiode. The static resistance $R(I)$ of a thermodiode in the considered model is identified with the magnitude

$$
R(I)=\frac{U}{I}=\frac{k T}{q I} \ln \left(\frac{I}{I_{s}}+1\right)
$$

where $I_{S}=J_{S} S$, and $U$ and $J_{S}$ are determined by equations (4) and (2), respectively.

The dynamic resistance $r(I)$ is equal to

$r(I)=\frac{d U}{d I}=\frac{k T}{q\left(I+I_{s}\right)}$.

\section{Limiting parameters of diode temperature sensors}

It follows from the above analysis that at some set of electrophysical and design parameters of the diode the maximum length of TMC is realized, and another set provides the maximum sensitivity of DTS. The procedure of their determination should be carried out using the self-consistent technique, since the parameters entering into these sets are interconnected. It is of interest to compare the values of these parameters for specific semiconductor materials, based on which the sensors with abrupt asymmetric $p n$-junction, where diffusion transport mechanism is realized, are fabricated. Below the numerical estimations of parameters are carried out for DTS fabricated from $\mathrm{Ge}, \mathrm{Si}$, and GaAs. The choice of these structures is explained by the fact that an industrial technology of diode manufacture based on these materials is well developed $[15,16]$.

The semiconductor constants necessary for the calculation are presented in Table 1. In calculations it is taken into account that in the case of full ionization of impurity atoms the minority carrier lifetime is independent of temperature [10]. For the calculation of the temperature dependence of mobility the relations used are presented in Table 2 . The total mobility was calculated using the Matissen rule with account of charge carrier scattering at ionized impurities and at acoustic (in the case of $\mathrm{Ge}, \mathrm{Si}$ ) or optical (in the case of 


\section{N. R. Kulish et al.: Self-consistent method for optimization of parameters ...}

Table 1. Constants of semiconductors used in calculations of limiting parameters of diode temperature sensors.

\begin{tabular}{cccc}
\hline \hline Parameter & $\mathrm{Ge}$ & $\mathrm{Si}$ & $\mathrm{GaAs}$ \\
\hline$E_{g}(T=0), \mathrm{eV}$ & $0.7412[10]$ & $1.1557[10]$ & $1.5216[10]$ \\
$a, \mathrm{eV} / \mathrm{K}$ & $4.774 \cdot 10^{-4}[8]$ & $4.73 \cdot 10^{-4}[8]$ & $5.405 \cdot 10^{-4}[8]$ \\
$b, \mathrm{~K}$ & $235[8]$ & $636[8]$ & $204[8]$ \\
$m_{e} / m_{o}$ & $0.56[10]$ & $1.08[10]$ & $0.068[10]$ \\
$m_{h} / m_{o}$ & $0.35[10]$ & $0.56[10]$ & $0.49[10]$ \\
\hline \hline
\end{tabular}

GaAs) phonons.

\subsection{The set of parameters providing the maximum length of $T M C$}

To achieve the maximum length of TMC towards a higher temperature range, the base region should be doped to the maximum possible level when the influence of the tunnel processes on transport can still be neglected. This situation occurs when $U_{b}>6 E_{g} / q$, i.e. when the breakdown is due to the avalanche multiplication only [8]. Therefore, for estimation of $N_{A m}$ (Table 3$)$ in (6) the value $U_{b}=6 E_{g}(300 \mathrm{~K}) / q$ should be used [8]. It should be noted that $U_{b}$ does not depend on the type of conductivity of the base [9], therefore, for $n$ - and $p$-types of conductivity the equation (6) gives the same values of the doping impurity concentration (Table 3). The emitter doping impurity concentration was assumed to be equal to the limit concentration of solubility of impurities commonly used in the diode fabrication technology $[15,16]$. To find the temperature $T_{m}=T_{i}{ }^{\prime}$, corresponding to the maximally possible length of TMC, one should insert into (9) the known values of $N_{A}=N_{A m}$ (Table 3) and of semiconductor constants (Table 1), and also to take into account the dependencies of $E_{g}$ and of the product $N_{c} N_{v}$ on temperature. The calculated values of $T_{m}=T_{i}{ }^{\prime}$ are presented in Table 3.

Since the operation current density, base length, $p n$-junction depth and minority carrier lifetime in the base are interrelated (see (5)), then for determination of the optimized (corresponding to the maximum length of TMC) values of these parameters we will carry out the following procedure. Let us take into account that in (8) the thermal conductivity coefficient $\lambda$ (Fig. 2) and the voltage drop $U$ across the $p n$-junction are functions of temperature. At a fixed value of $\Delta T_{H}$ and $l$ one should insert into (8) the maximum value of the voltage drop at $p n$-junction $U=U_{\max }$ and the minimum value

Table 2. Equations used in calculation of carrier mobility.

\begin{tabular}{|c|c|c|c|}
\hline Semiconductor & $\begin{array}{l}\text { Type of } \\
\text { conduc- } \\
\text { tivity }\end{array}$ & $\begin{array}{c}\mu_{L}^{*} \\
\mathrm{~cm}^{2} / \mathrm{V} \mathrm{s}\end{array}$ & $\begin{array}{c}\mu_{I}^{*} \\
\mathrm{~cm}^{2 / V ~ s}\end{array}$ \\
\hline \multirow[t]{2}{*}{$\mathrm{Ge}$} & $n$ & $\mu_{L n}={\frac{4,9 \cdot 10^{7}}{T^{1,66}}}^{7}$ & $\mu_{I n}=\frac{11,4 \cdot 10^{17} \cdot T^{1,5}}{N \ln \left(1+8,3 \cdot 10^{8} \cdot T^{2} / N^{2 / 3}\right)}$ \\
\hline & $p$ & $\mu_{L p}=\frac{1,05 \cdot 10^{9}}{T^{2,33}}$ & $\mu_{I p}=\frac{14,2 \cdot 10^{17} \cdot T^{1,5}}{N \ln \left(1+8,3 \cdot 10^{8} \cdot T^{2} / N^{2 / 3}\right)}$ \\
\hline \multirow[t]{2}{*}{$\mathrm{Si}$} & $n$ & $\mu_{L n}={\frac{3,58 \cdot 10^{9}}{T^{2,6}}}^{9}$ & $\mu_{I n}=\frac{4,7 \cdot 10^{17} \cdot T^{1,5}}{N \ln \left(1+4,5 \cdot 10^{8} \cdot T^{2} / N^{2 / 3}\right)}$ \\
\hline & $p$ & $\mu_{L p}=\frac{2,40 \cdot 10^{8}}{T^{2,3}}$ & $\mu_{I p}=\frac{5,8 \cdot 10^{17} \cdot T^{1,5}}{N \ln \left(1+4,5 \cdot 10^{8} \cdot T^{2} / N^{2 / 3}\right)}$ \\
\hline \multirow[t]{2}{*}{ GaAs } & $n$ & $\mu_{L n}={\frac{1,58 \cdot 10^{5}}{T^{0,5}}}^{5}$ & $\mu_{I n}=\frac{20,7 \cdot 10^{17} \cdot T^{1,5}}{N \ln \left(1+5,4 \cdot 10^{8} \cdot T^{2} / N^{2 / 3}\right)}$ \\
\hline & $p$ & $\mu_{L p}=\frac{9,62 \cdot 10^{3}}{T^{0,5}}$ & $\mu_{I p}=\frac{8,2 \cdot 10^{17} \cdot T^{1,5}}{N \ln \left(1+5,4 \cdot 10^{8} \cdot T^{2} / N^{2 / 3}\right)}$ \\
\hline
\end{tabular}

*Mobility due to phonon scattering $\mu_{L}$ and scattering at ionized impurity atoms $\mu_{\boldsymbol{I}}$. 


\section{N. R. Kulish et al.: Self-consistent method for optimization of parameters ...}

Table 3. Limiting parameters of temperature sensors with the maximum length of thermometric characteristic

\begin{tabular}{llll}
\hline \hline Parameter & $\mathrm{Ge}$ & \multicolumn{1}{c}{$\mathrm{Si}$} & $\mathrm{GaAs}$ \\
\hline$U_{b}, \mathrm{~W}$ & 4.45 & 6.93 & 9.13 \\
$N_{D}, N_{A}, \mathrm{~cm}^{-3}$ & $1.35 \cdot 10^{17}$ & $1.90 \cdot 10^{17}$ & $2.25 \cdot 10^{17}$ \\
$T_{m}, \mathrm{~K}$ & 484 & 703 & 995 \\
$\lambda\left(T_{m}\right), \mathrm{W} / \mathrm{cm} \cdot \mathrm{K}$ & 0.30 & 0.50 & 0.15 \\
$\Delta T_{0}, \mathrm{~K}(d=l=3 \mu)$ & $6 \cdot 10^{-2}$ & $1 \cdot 10^{-2}$ & $4 \cdot 10^{-1}$ \\
$J_{\operatorname{mo}}, \mathrm{A} / \mathrm{cm}^{2}$ & 85.35 & 17.00 & 186.00 \\
$I_{\max }, \mathrm{A}$ & $7.68 \cdot 10^{-2}$ & $1.53 \cdot 10^{-2}$ & $1.67 \cdot 10^{-1}$ \\
$S_{\max }, \mathrm{cm}^{2}$ & $9 \cdot 10^{-4}$ & $9 \cdot 10^{-4}$ & $9 \cdot 10^{-4}$ \\
$\alpha\left(T=T_{m}\right), \mathrm{V} / \mathrm{K}$ & $-6.8 \cdot 10^{-4}$ & $-8.0 \cdot 10^{-4}$ & $-6.0 \cdot 10^{-4}$ \\
\hline$\tau_{n}, \mathrm{~s}$ & & $\boldsymbol{n}^{+} \boldsymbol{p}-\mathrm{junction}$ & $1.3 \cdot 10^{-9}$ \\
$I_{\min }, \mathrm{A}$ & $2.1 \cdot 10^{-8}$ & $5.2 \cdot 10^{-6}$ \\
$S_{\min }, \mathrm{cm}^{2}$ & $5.3 \cdot 10^{-9}$ & $1.4 \cdot 10^{-4}$ & $2.8 \cdot 10^{-8}$ \\
\hline & $1.4 \cdot 10^{-5}$ & $8.0 \cdot 10^{-6}$ & \\
$\tau_{p}, \mathrm{~S}$ & $1.7 \cdot 10^{-7}$ & $\boldsymbol{p}^{+} \boldsymbol{n}-\mathrm{junction}$ & $2.4 \cdot 10^{-11}$ \\
$I_{\min }, \mathrm{A}$ & & $4.0 \cdot 10^{-9}$ & $2.8 \cdot 10^{-4}$ \\
$S_{\min }, \mathrm{cm}^{2}$ & $7.4 \cdot 10^{-4}$ & $1.5 \cdot 10^{-6}$ \\
\hline \hline
\end{tabular}

of thermal conductivity coefficient $\lambda=\lambda_{\min }\left(\right.$ at $\left.T \leq T_{m}\right)$. In this case at any temperature registered by the diode sensor in the considered temperature range the Joule heating will not exceed $\Delta T_{H}$. If, for estimation of $J_{m}$, we assume that $U_{\max }=E_{g}\left(T_{m}\right) / q$, then, with account of above arguments, (8) is converted to the form

$$
J_{m}=\frac{\Delta T_{H} q \lambda_{\min }}{l E_{g}\left(T_{m}\right)},
$$

and the equation (4) is written as follows

$$
U(T)=\frac{k T}{q} \ln \left(\frac{J_{m}}{J_{s}(T)}+1\right)
$$

The equation (24) describes the parametric set of TMC crossing the point $\left(U\left(T_{m}\right), T_{m}\right)$ in the plane $(U, T)$. In this set every individual characteristic is determined by its own number of parameters $\Delta T_{H}, d, l, \tau_{n}$. It is most easy to establish connection between these parameters in the limiting point $T=T_{m}$. Suppose that the current density in (5) is equal to $J_{m}$ determined according to (23). Then instead of (5) we can write

$$
\Delta T_{H}=\Delta T_{0} \frac{l}{L_{n}\left(T_{m}\right)} \operatorname{coth} \frac{d}{L_{n}\left(T_{m}\right)},
$$

where

$$
\Delta T_{0}=\frac{(e-1) \mu_{n}\left(T_{m}\right) k T_{m} n_{i}^{2}\left(T_{m}\right) E_{g}\left(T_{m}\right)}{q \lambda_{\min } N_{A}} .
$$

For the case $d=l$ (see Fig. 1a) in Fig. 3 the dependencies are shown of the temperature measurement systematic error $\Delta T_{H}$ on the ratio $d / L_{n}\left(\mathrm{~T}_{m}\right)$ calculated according to $(25)$ for $\mathrm{Ge}-, \mathrm{Si}$ and GaAs-based diode temperature sensors. It can be seen in Fig. 3 that with decreasing ratio $d / L_{n}\left(T_{m}\right)$ the reduction of $\Delta T_{H}$ is observed with gradual approaching to the limiting value $\Delta T_{0}$. Values $\Delta T_{0}$ calculated from (26) for $\mathrm{Ge}-, \mathrm{Si}-$ and GaAs-based diode temperature sensors are presented in Table 3 .

It follows from (25) that at $\mathrm{d} \neq l$ in the short-base diodes $\left(d<<L_{n}\right) \Delta T_{H}=(l / d) \Delta T_{0}$. It is clear that for a fixed value $l / d$

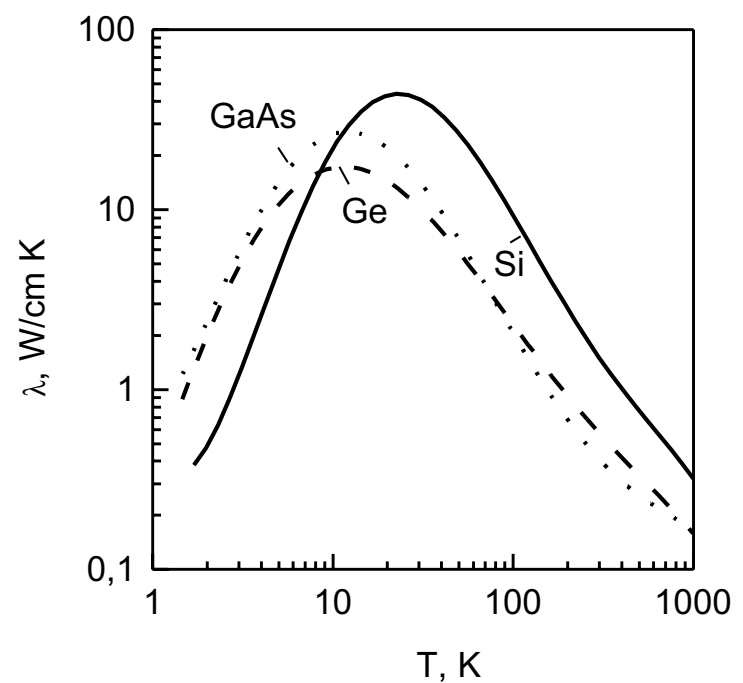

Fig. 2. Temperature dependence of thermal conductivity $\lambda(T) \mathrm{Ge}$ $[8,17]$, Si $[8,17]$, GaAs $[8,18]$. 


\section{N. R. Kulish et al.: Self-consistent method for optimization of parameters ...}

there is a range of $d / L_{n}\left(T_{m}\right)$ variation, within which $\Delta T_{H}$ varies slightly (Fig. 3). It should be noted that $\Delta T_{H}$ is less than $\Delta T_{\mathrm{o}}$, if $l / d<1$. Inserting into (23) the value $\Delta T_{H}$ from (25) we get the current density $J_{m}$ for the short-base diode as follows

$J_{m}=J_{m o} \equiv \frac{(e-1) \mu_{n}\left(T_{m}\right) k T_{m} n_{i}^{2}\left(T_{m}\right)}{N_{A} d}$.

It follows from (27), with account of (9), that in the shortbase diodes the increase of $N_{A}$ and decrease of $\mathrm{d}$ result in an increase of $J_{m}$. Values $J_{m}=J_{m o}$ calculated from (27) using data of Tables 1-3 are presented in Table 3.

Similarly it follows from (25) that in the long-base diodes $\left(d>>L_{n}\left(T_{m}\right)\right) \Delta T_{H}=\Delta T_{0}\left(l / L_{n}\left(T_{m}\right)\right)$. If, in this case also $l=d$ (Fig. 1a), then $\Delta T_{H}=\Delta T_{0}\left(d / L_{n}\left(T_{m}\right)\right)$. The current density $J_{m}$ in the long-base diodes can be calculated using the relation

$J_{m}=J_{m o}\left(d / L_{n}\left(T_{m}\right)\right)$,

where $J_{m o}$ is calculated from (27). It follows from (28) that in the long-base diodes the characteristic current density $J_{m}$ is independent of the base length $d$, if just this magnitude determines the distance from the pn-junction to the cooling surface (Fig. 1a).

The above relations make it possible to give following recommendations regarding the choice of chip design and pn-junction parameters for DTS. The metallurgic boundary of the pn-junction in diode structures fabricated using diffusion and/or ion-implantation techniques used to be situated at a depth ranging from 0.3 to $15 \mu \mathrm{m}$ in respect to the crystal face $[15,16]$. To reduce the effect of the surface on the transport current through the $p n$-junction it is usually situated at a depth of $2-3 \mu \mathrm{m}$. To reduce the

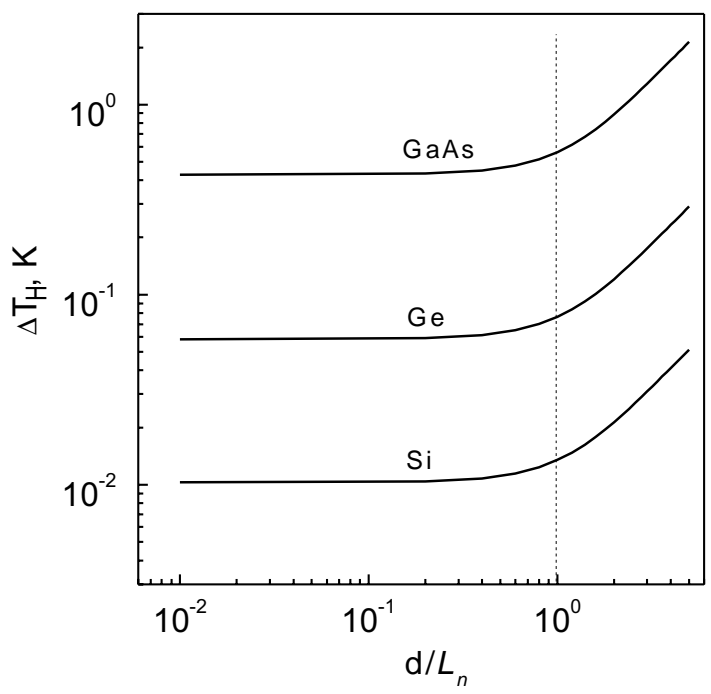

Fig. 3. Dependence of the systematic error $\Delta T_{H}$ of temperature measurement by Ge, Si, GaAs diode sensor on the base length $d$, normalized by the diffusion length $L_{n}$ of minority charge carriers. Calculation was performed for $T=T_{m}$. Dashed line indicates the value $d / L_{n}=1$. effect of bondage of the semiconductor chip to the basis of the package on the transport current in diode structures, $p n$ junction is usually situated in the top part of the chip (Fig. 1b). From the viewpoint of using the diode structure as the temperature sensor such a construction of the chip is the least reasonable, because in this case $l$ is much longer than $d$, which, according to (25) results in the greater temperature measurement systematic error $\Delta T_{H}$ due to Joule heating of the pnjunction.

The minimum value of $\Delta T_{H}$ is realized in the construction of the short-base diode sensor shown in Fig.1c. If the thin emitter layer, with thickness less than the base length, is present in this construction, $\Delta T_{H}$ can be less than $\Delta T_{0}$. The systematic error of the temperature measurement by the diode sensor, the construction of which is presented in Fig.1c, will be significantly less than the systematic error of the temperature measurement by the sensor shown in Fig. 1b. Thus, in the specific technical realization of the diode temperature sensor the preference should be given to the short-base diode structures with reverse mounting of the chip on the package basis. For these structures at $d=L_{n}\left(T_{m}\right)$ the connection between the base length and the minority carrier lifetime in the base is determined by the relation

$d=L_{n}\left(T_{m}\right)=\sqrt{\frac{k T_{m} \mu_{n}\left(T_{m}\right) \tau_{n}}{q}}$.

According to Fig. 3, $\Delta T_{H}$ slightly depends on the ratio $d / L_{n}$ up to the value $d / L_{n}=1$. Note that the typical pnjunction depth $\mathrm{d}=(2-3) \mu \mathrm{m}$. Assuming, for the definiteness, $d=L_{n}\left(T_{m}\right)=3 \mu \mathrm{m}$, from (29) we calculate the minority carrier lifetime (Table 3 ).

To find the value of sensitivity (Table 3 ) at the point $T=T_{m}$ we will use the relation (13). The minimum value of the operation current is found from (17). First, inserting into (18) $T=T_{m}, \tau_{n}$ and $\alpha\left(T_{m}\right)$ from Table 3 we calculate $I_{0}$, and also the value $\Delta T_{N}$, assuming $\Delta T_{N}=\Delta T_{H}$. Then we express $I_{s}$ via $I\left(I=I_{\min }\right)$ using (12). Then we insert the values of $I_{S}$ and $I_{0}$ into (17) and solve the obtained equation in respect to $I_{\min }$ (Table 3 ).

To estimate $S_{\min }$ and $I_{\max }$ we will use the equations (19) and (20) and the parameters from Table 3.

Thus, the calculation carried out in the section 2.1 permits to determine the set of parameter values providing the achievement of the maximum extent of the TMC into the higher temperature region. For $\mathrm{Ge}-, \mathrm{Si}-$ and GaAs-based DTS with asymmetric $n^{+} p$ - or $p^{+} n$-junction these parameters are presented in Table 3.

\subsection{Set of parameters providing the maximum sen- sitivity of diode temperature sensors}

From a number of similar DTS the sensors with the maximum sensitivity are preferable. Let us investigate the behaviour of $\alpha(T)$ at variation of temperature and parameters of the 


\section{N. R. Kulish et al.: Self-consistent method for optimization of parameters ...}

structure.

At the upper boundary of the temperature range covered by the thermometric characteristic (4), $\alpha\left(T_{m}\right)$ is determined by the equation (13). At temperatures $T<T_{m}$ the sensitivity $\alpha(T)$ is higher than $\alpha\left(T_{m}\right)$ and slightly depends on temperature, except the relatively narrow transition region in the vicinity of $\mathrm{T}_{\mathrm{m}}$, where it falls down to its limiting value (13). In the plateau region, i.e. out of the mentioned transition region, the value $\alpha$ is determined by the equation (11).

At $\tau_{n} \gg d^{2} /\left(k T \mu_{n} / q\right) \equiv \tau_{D}$ the situation of the short-base diode is realized $\left(d<<L_{n}\right)$ when

$$
\alpha(T)=-\frac{k}{q} \ln \left\{\frac{4 S\left(2 \pi k T / h^{2}\right)^{3}\left(m_{e} m_{h}\right)^{3 / 2} \mu_{n} k T}{I N_{A} d}\right\} .
$$

Thus, in the case of the short-base diode, sensitivity of the sensor is independent of $\tau_{n}$ unless the characteristic minority carrier lifetime in the base is essentially longer than the characteristic time $\tau_{D}$ of carrier diffusion to the Ohmic contact where the surface recombination rate is infinite.

With reduction of $\tau_{n}$ (at $\tau_{n}<<\tau_{D}$ ) another limiting case of the long-base diode $\left(d>>L_{n}\right)$ is realized for which sensitivity $\alpha(T)$ is obtained from (30) by substitution of $d$ by $L_{n}$

$\alpha(T)=-\frac{k}{q} \ln \left\{\frac{4 S\left(q \mu_{n} k T\right)^{1 / 2}\left(2 \pi k T / h^{2}\right)^{3}\left(m_{e} m_{h}\right)^{3 / 2}}{I N_{A} \tau_{n}^{1 / 2}}\right\}$.

With account of restrictions imposed on the base resistance we find the criterion of validity of the equation (31) at the increasing base length in the form $L_{n}<<d<<d_{b} \equiv q \mu_{p} N_{A} S R(I)$, where $R(I)$ is the resistance of the pn-junction $\left(\mathrm{d}_{\mathrm{b}}\right.$ has the sense of the length of the base with the resistance comparable with that of the pn-junction resistance).

According to (30), (31), higher sensitivity is provided at the larger $p n$-junction area $S$, lower current $I$, concentration $N_{A}$ and shorter base length $d$ (for the short-base diodes) or minority carrier lifetime $\tau_{n}$ in the base (for the long-base diodes).

In calculation of $\alpha(T)$ we will assume that the maximum value of the $p n$-junction area $\left(S=S_{\max }\right)$ (Table 4$)$ is limited from above by the technology of wafer sawing into chips of minimum possible size (see section 2.1).

The minority carrier lifetime in the base of diodes fabricated on Czochralski-grown $\mathrm{Ge}, \mathrm{Si}$ and $\mathrm{GaAs}$ single crystals is about $10^{-8} \mathrm{~s}[15,16,19]$. By reduction of concentration of oxygen precipitates it can be increased to $10^{-5} \mathrm{~s}[7,19]$, and by heavy doping with impurities generating deep centres it is possible to reduce it to few picoseconds [20-22]. With increasing the doping concentration of deep impurities $N_{I}>10^{17} \mathrm{~cm}^{-3}$ (corresponding to the minority carrier lifetime $\tau_{n} \approx 10^{-9} \mathrm{~s}$ ) the reduction of $\tau_{n}$ in Ge, Si [23] and GaAs [22] is accompanied with the reduction of the charge carrier mobility. At high concentrations $\mathrm{N}_{\mathrm{I}}$ of charged deep centres the mobility $\mu_{n} \approx \mu_{I} \sim N_{I}^{-1}$ and lifetime $\tau_{n} \sim N_{I}^{-1}$. Therefore, in the shortbase diodes an increase of the deep centre concentration results in reduction of $\alpha(T)$ (30) due to mobility reduction, and in the long-base diodes the value of $\alpha(T)$ (31) should not vary significantly, because its reduction due to a decrease of $\tau_{n}$ is compensated by the respective mobility reduction. Below, in calculation of $\alpha(T)$, it is assumed that the average minority carrier lifetime $\tau_{n}=\tau_{p}=10^{-9} \mathrm{~s}$ (Table 4), which corresponds to $L_{n}>d=1 \mu \mathrm{m}$ (Table 4 ).

Table 4. Limiting parameters of the temperature sensors with the maximum sensitivity.

\begin{tabular}{lccc}
\hline Parameter & $\mathrm{Ge}$ & $\mathrm{Si}$ & $\mathrm{GaAs}$ \\
\hline$N_{D}, N_{A}, \mathrm{~cm}^{-3}$ & $1.0 \cdot 10^{14}$ & $1.0 \cdot 10^{14}$ & $1.0 \cdot 10^{14}$ \\
$\tau_{n}=\tau_{p}, \mathrm{~s}$ & $1.0 \cdot 10^{-9}$ & $1.0 \cdot 10^{-9}$ & $1.0 \cdot 10^{-9}$ \\
$S_{\max }, \mathrm{cm}^{2}$ & $9 \cdot 10^{-4}$ & $910^{-4}$ & $910^{-4}$ \\
& & & \\
& & $n^{+} p$ - junction & 513 \\
$T_{m}, \mathrm{~K}$ & 260 & 390 & 53.2 \\
$\Delta T\left(T=T_{m}\right), \mathrm{mK}$ & 36.5 & 89.7 & 2.36 \\
$\alpha(T=300 \mathrm{~K}), \mathrm{mW} / \mathrm{K}$ & 1.74 & 2.72 & 17.8 \\
$J, \mathrm{~mA} / \mathrm{cm}^{2}$ & 9.6 & 3.0 & 16.0 \\
$I, \mu \mathrm{A}$ & 8.6 & 2.7 & \\
\hline
\end{tabular}

$$
p^{+} n \text { - junction }
$$

$\begin{array}{lccc}T_{m}, \mathrm{~K} & 262 & 394 & 526 \\ \Delta T\left(T=T_{m}\right), \mathrm{mK} & 45.5 & 111.3 & 129.0 \\ \alpha(T=300 \mathrm{~K}), \mathrm{mW} / \mathrm{K} & 1.76 & 2.67 & 2.26 \\ J, \mathrm{~mA} / \mathrm{cm}^{2} & 6.3 & 2.0 & 3.3 \\ I, \mu \mathrm{A} & 5.7 & 1.8 & 3.0\end{array}$




\section{N. R. Kulish et al.: Self-consistent method for optimization of parameters ...}

Further increase of sensitivity (see (30), (31)) can be provided by the reduction of the current I and of doping impurity concentration $N_{A}$ in the base. It should be mentioned, however, that the range of variation of $N_{A}$ is limited from above by the concentration corresponding to the case when the effect of tunnel processes on transport in the diode structure can still be neglected, and is limited from below by the concentration of uncontrollable impurities in the semiconductor, the value of which for $\mathrm{Ge}, \mathrm{Si}$ and $\mathrm{GaAs}$ is taken to be equal to $10^{14} \mathrm{~cm}^{-3}$ (Table 4) $[15,16]$.

Since the base resistance increases with reduction of $N_{A}$, then the process of temperature measurement can be affected, in addition to systematic errors due to Joule heating of $p n$-junction $\Delta T_{H}$ (see (25)) and the presence of noise $\Delta T_{N}$ (see (16), (15)), by the systematic error $\Delta T_{R}$, appearing as a result of an increase of the base resistance

$\Delta T_{R}=R_{b}\left(T, N_{A}\right) I /\left|\alpha\left(T, N_{A}\right)\right|$,

where the base resistance $R_{b}\left(T, N_{A}\right)=\left(d / q \mu_{p} N_{A} S\right)[1+$ $\left.+\left(\mu_{p} / \mu_{n}-1\right)\left(L_{n} / d\right) \operatorname{th}\left(d / L_{n}\right)\right][6]$.

Because in the considered model the effect of the base resistance on TMC and sensitivity is neglected, then the magnitude of $\alpha(T)$ entering into (32) and (16) is determined from the variation of the voltage drop across the $p n$-junction. The total systematic error is equal to the sum of the above mentioned components. It follows from (5) and (27), (28), that the decrease of $N_{A}$ results in reduction of the limiting temperature $T_{m}$, which becomes less than $T_{i}^{\prime}$, of the current density $J_{m}$ and, according to (7), of $\Delta T_{H}$. Therefore, in the further analysis it is sufficient to restrict ourselves by the total error

$\Delta T=\Delta T_{N}+\Delta T_{R}$

Let us insert the values $d=1 \mu \mathrm{m}$ and $\tau_{n, p}, S_{\max }$ from Table 4 , and the current density from (12) into (4) and (33). In this case both the equation of thermometric characteristic (4) and the equation of systematic error (33) becomes the twoparameter ones, depending on $T_{m}$ and $N_{A}$. At a fixed value of temperature $T_{m}$, the dependence $\Delta T$ on $N_{A}$, resulting from (33) has the minimum $\Delta T_{\min }=\Delta T\left(N_{A o}\right)$ at some value of doping impurity concentration $N_{A}=N_{A o}$ (Fig. 4). The concentration $N_{A}=N_{A o}$, corresponding to $\Delta T=\Delta T_{\text {min }}$, is then taken as a required doping impurity concentration which will be used for the calculation of TMC of the diode sensor from the mentioned set of thermometric characteristics

$$
U(T)=\frac{k T}{q} \ln \left[\frac{(e-1) J_{S}\left(T_{m}, N_{A o}\right)}{J_{S}\left(T, N_{A o}\right)}+1\right] .
$$

At a given temperature $T_{m}\left(T_{m}<T_{i}\right)$ we calculate $N_{A o}\left(T_{m}\right)$ and then determine the current density $\mathrm{J}$ from (12) and the operation current from the relation $I=J S_{\max }$. These parameters, calculated for minimum acceptable concentration of doping impurity in the base $N_{A o}=10^{14} \mathrm{~cm}^{-3}$ for Ge, Si and GaAs
DTS, are presented in Table 4. The above self-consistent procedure enabled to calculate the apparent dependence of the doping impurity concentration $N_{A o}$ in the base on $T_{m}$ (Fig.5, bold lines), and to determine also the set of parameters $J, d / L_{n}, \tau_{n}, N_{A o}$, providing the highest sensitivity of the sensor. In particular, inserting into (11) values of these parameters and the magnitude $\mu_{n}$ for temperature $300 \mathrm{~K}$, we find the dependence of sensitivity $\alpha(T=300 \mathrm{~K})$ on the impurity concentration $N_{A o}$ (Fig. 5, thin lines). It can be seen in Fig. 5 that with increasing $N_{A o}$ the extent of TMC increases and sensitivity of DTS decreases.

\section{Limiting characteristics of diode temperature sensors}

Tables 3 and 4 contain the set of parameters, insertion of which into equations presented in sections 1 and 2 makes it possible to find the limiting characteristics of $\mathrm{Ge}, \mathrm{Si}$, GaAs DTS: the temperature dependencies of the voltage drop across the $p n$-junction (TMC) (Fig. 6), sensitivity (Fig. 7), static (Fig. 8) and dynamic (Fig. 9) resistances for $n^{+} p$ and $p^{+} n$-junction-type diode temperature sensors.

In figures 6-9 numerals denote the characteristics measured experimentally; letter $\mathrm{r}$ denotes the characteristics calculated using the parameter set providing the maximum length of TMC (Table 3); 1 indicate those calculated using a set providing maximum sensitivity (Table 4). Thin solid line in the Fig. 6 shows the temperature dependence of the thermal voltage $U_{T}=k T / q$. Curves denoted by letter $\mathrm{r}$ and 1 determine the boundaries within which the characteristics of DTS can vary during variation of electrophysical and design parameters of the diode. From comparison of data presented in figures $6 a-9 a$ and figures $6 b-9 b$ one can see that a change of base conductivity from $p$-type to $n$-type has no marked effect on the position of these boundaries. It follows from figures 6-9 that experimentally measured characteristics of

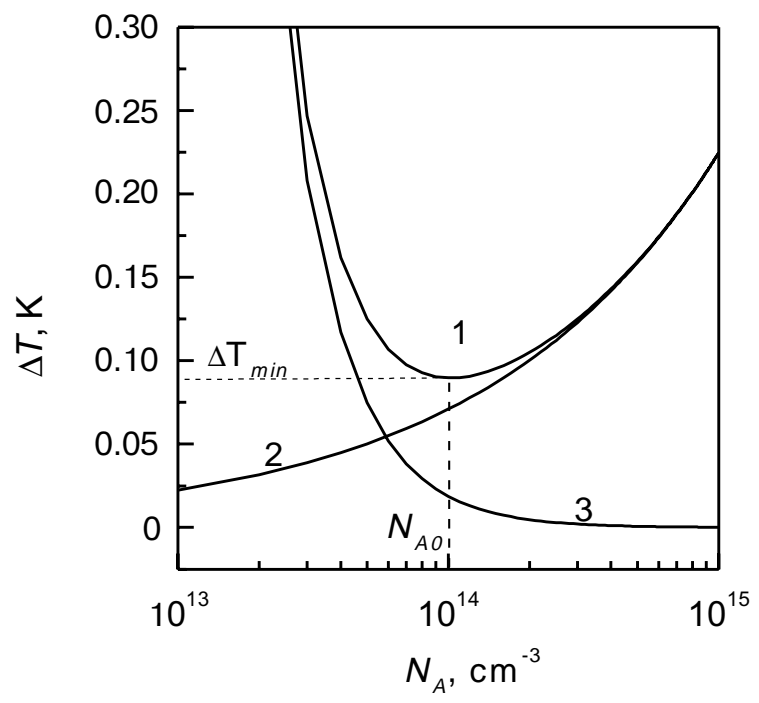

Fig. 4. Dependence of the systematic error $\Delta T$ (1), due to both noise (2), and the base resistance (3), on the impurity concentration in the base. $T_{m}=300 \mathrm{~K}$. 


\section{N. R. Kulish et al.: Self-consistent method for optimization of parameters ...}

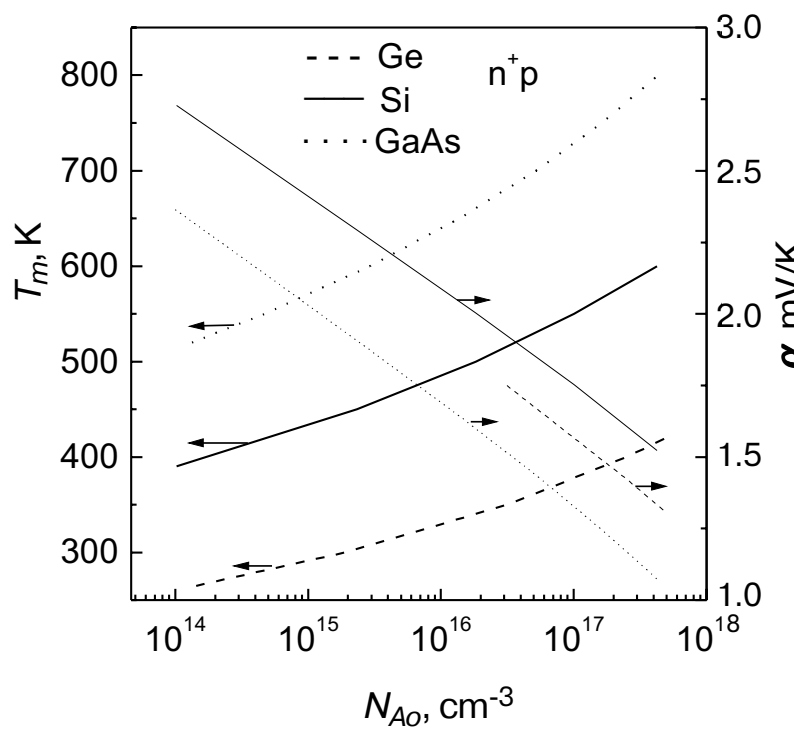

a

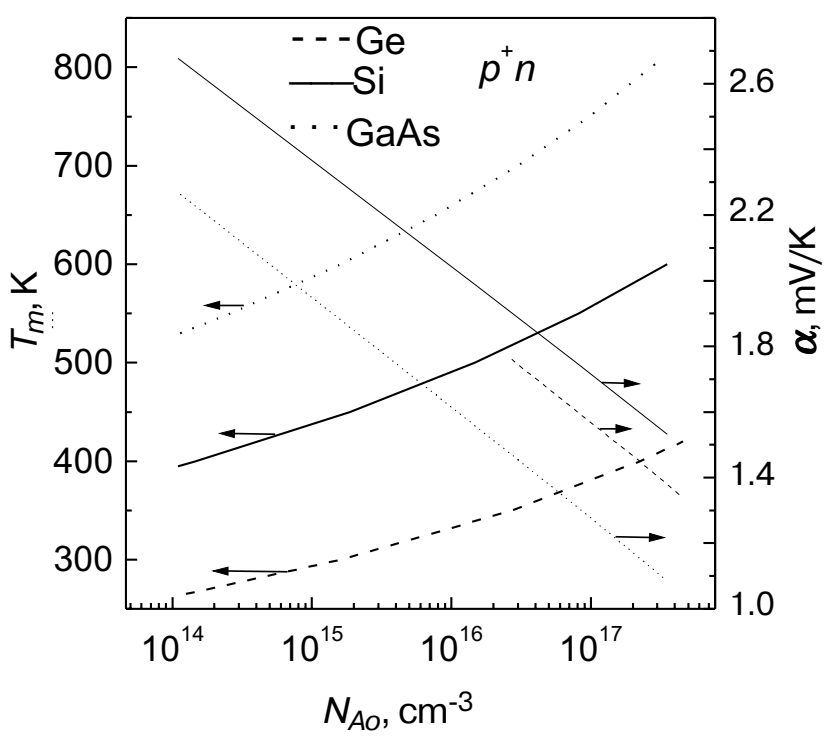

b

Fig. 5. Dependencies on the impurity concentration $N_{A o}$ in the base of $n^{+} p(a)$ и $p^{+} n(b)$ DTS of sensitivity at $T=300 \mathrm{~K}$ (thin lines) and of temperature $T_{m}$ (bold lines), determined by the aggregate of parameters providing the minimum error of temperature measurement calculated from the equation (33).

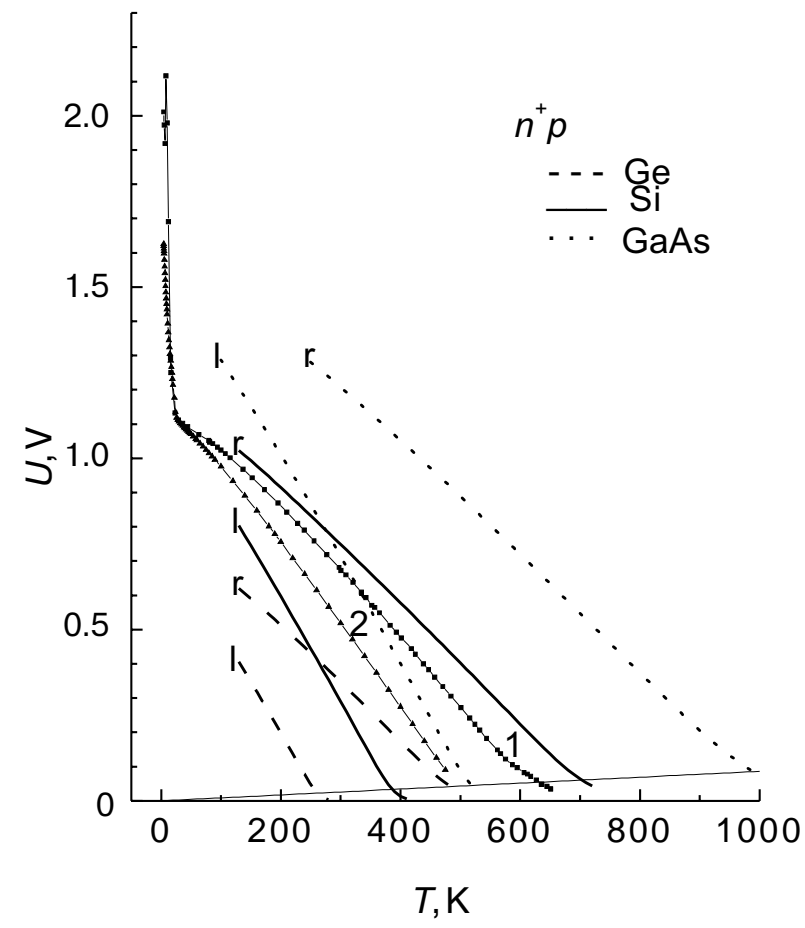

a

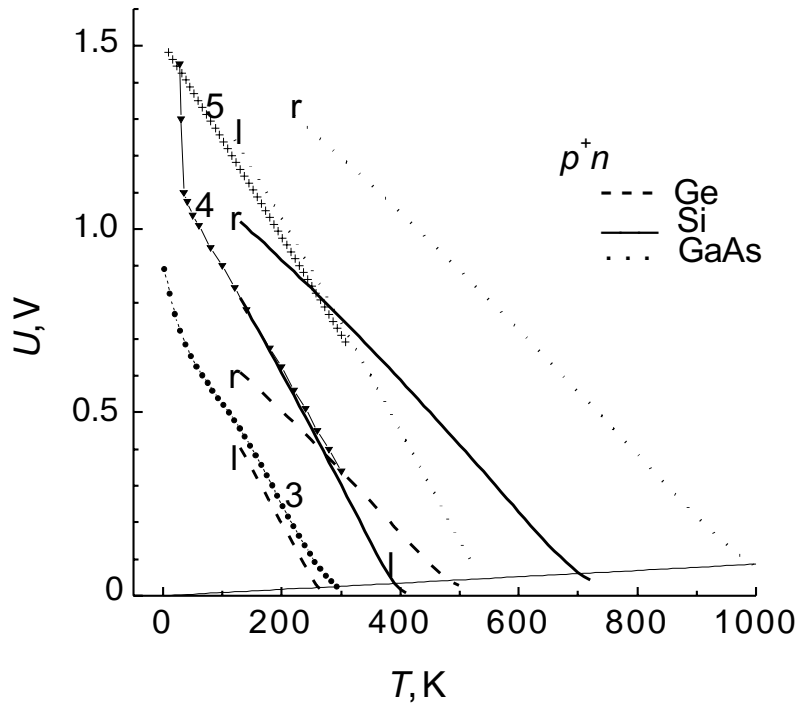

$\mathrm{b}$

Fig. 6. Thermometric characteristics of $\mathrm{Ge}, \mathrm{Si}, \mathrm{GaAs}$ diode sensors with abrupt asymmetric $n^{+} p$ (a) и $p^{+} n$ (b) junctions. Legends in the figure: $r$ - calculation using the parameter set providing the maximum length of TMC; 1 - calculation using the parameter set providing the maximum sensitivity of DTS; experimentally measured characteristics are denoted as: 1 - our data for the silicon temperature sensor $(I=10 \mu$, $\left.N_{A}=2 \times 10^{17} \mathrm{~cm}^{-3}\right), 2$ - data of [13] for the silicon temperature sensor $(I=10 \mu \mathrm{A}), 3$ - data of [2] for germanium temperature sensor $(I=10 \mu \mathrm{A}$, $\left.N_{A}=(1-2) \times 10^{17} \mathrm{~cm}^{-3}\right), 4$ - data of [1] for the silicon temperature sensor $\left(I=10 \mu \mathrm{A}, N_{A}=1.6 \times 10^{17} \mathrm{~cm}^{-3}\right), 5-$ data of [2] for GaAs temperature sensor $\left(I=10 \mu \mathrm{A}, N_{A}=9 \times 10^{17} \mathrm{~cm}^{-3}\right)$. 


\section{N. R. Kulish et al.: Self-consistent method for optimization of parameters ...}

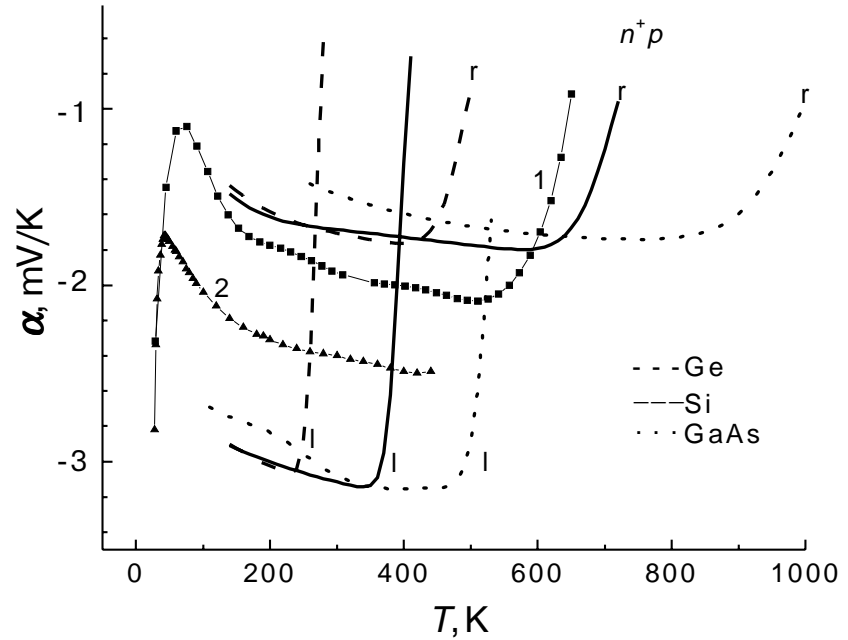

a

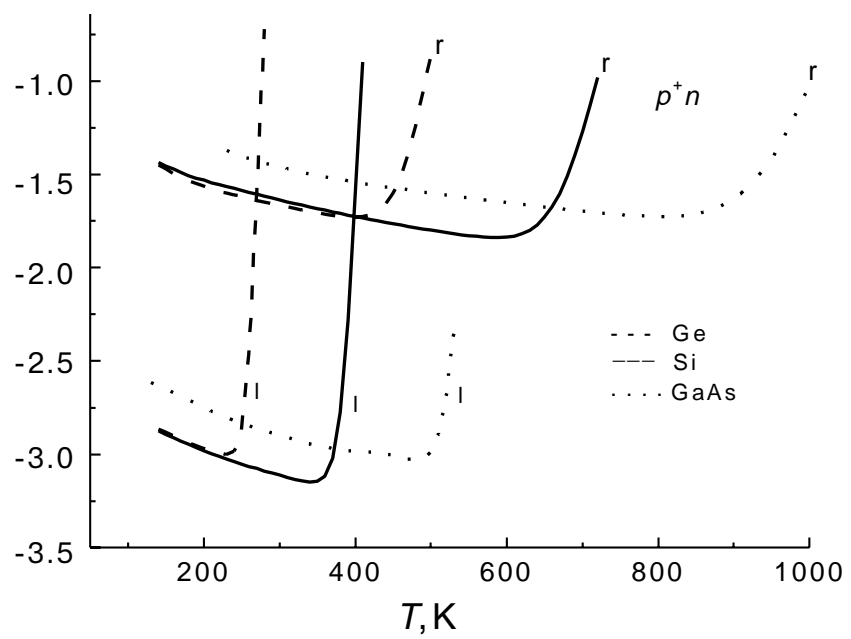

b

Fig. 7. Temperature dependencies of sensitivity exhibited by Ge, $\mathrm{Si}, \mathrm{GaAs}$ diode sensors with abrupt asymmetric $n^{+} p$ (a) и $p^{+} n$ (b) junctions. Legends in the figure: $r$ - calculation using the parameter set providing the maximum length of TMC; 1 - calculation using the parameter set providing the maximum sensitivity of DTS; experimentally measured characteristics are denoted as: 1 - our data for the silicon temperature sensor $\left(I=10 \mu \mathrm{A}, N_{A}=2 \times 10^{17} \mathrm{~cm}^{-3}\right), 2-$ data of [13] for the silicon temperature sensor $(I=10 \mu \mathrm{A})$.

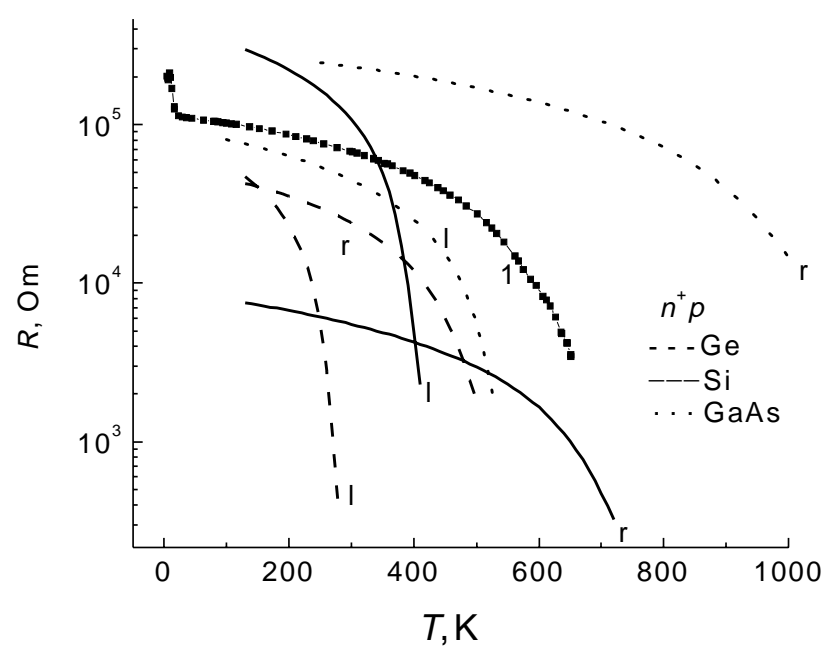

a

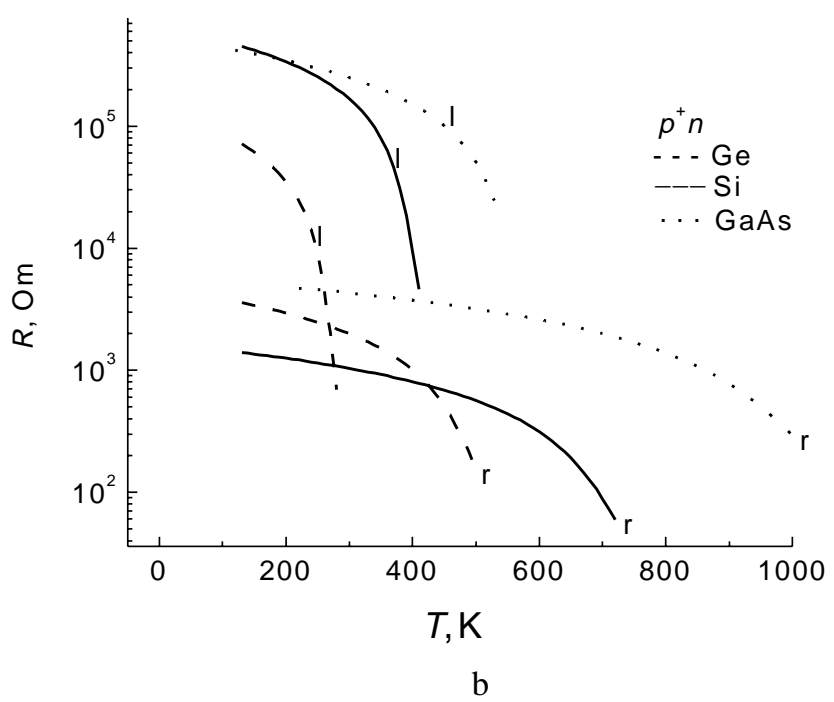

Fig. 8. Temperature dependencies of static resistance of Ge, Si, GaAs DTS with abrupt asymmetric $n^{+} p$ (a) и $p^{+} n$ (b) junctions. Legends in the figure: $r$ - calculation using the parameter set providing the maximum length of TMC; 1 - calculation using the parameter set providing the maximum sensitivity of DTS: $1-$ our data for the silicon temperature sensor $\left(I=10 \mu \mathrm{A}, N_{A}==2 \times 10^{17} \mathrm{~cm}^{-3}\right)$.

Ge and Si DTS lie within the mentioned boundaries. TMC of GaAs diode temperature sensor (Fig. 6b, curve 5), however, is located near the left boundary of the range (near the curve 1 in Fig. 6b). The impurity concentration in the base of this diode $\left(N_{D}=9 \times 10^{17} \mathrm{~cm}^{-3}\right)$ is much higher than the concentration $\left(N_{D}=2,25 \times 10^{17} \mathrm{~cm}^{-3}\right)$ used in calculation of the right boundary of the range. In the case of the diffusion-controlled transport current the experimental TMC should be situated near the right boundary (near the curve $r$ in the Fig.
$6 b)$. Its location near the left boundary indicates that other transport mechanisms dominate in this sensor.

Let us note that the magnitude of sensitivity $\alpha(T=300 \mathrm{~K})$ in Fig. 5 was calculated using the simplified equation (11), and the value of $\alpha$ indicated in Figure 7 was evaluated from the general equation (10). Comparison of these data has shown that the sensitivity evaluation using the simplified equation (11) gives the underestimated by $10-15 \%$ value. 


\section{N. R. Kulish et al.: Self-consistent method for optimization of parameters ...}

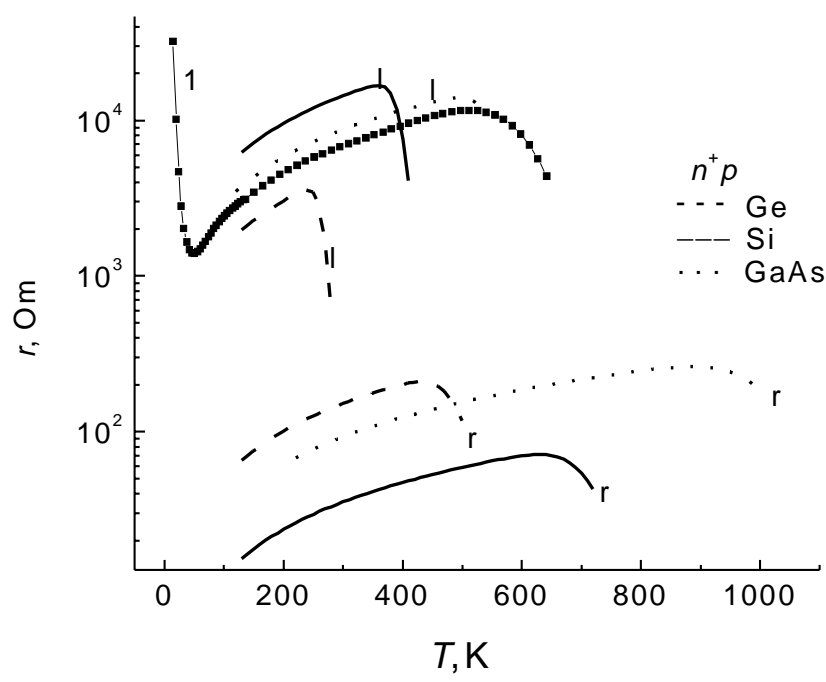

a

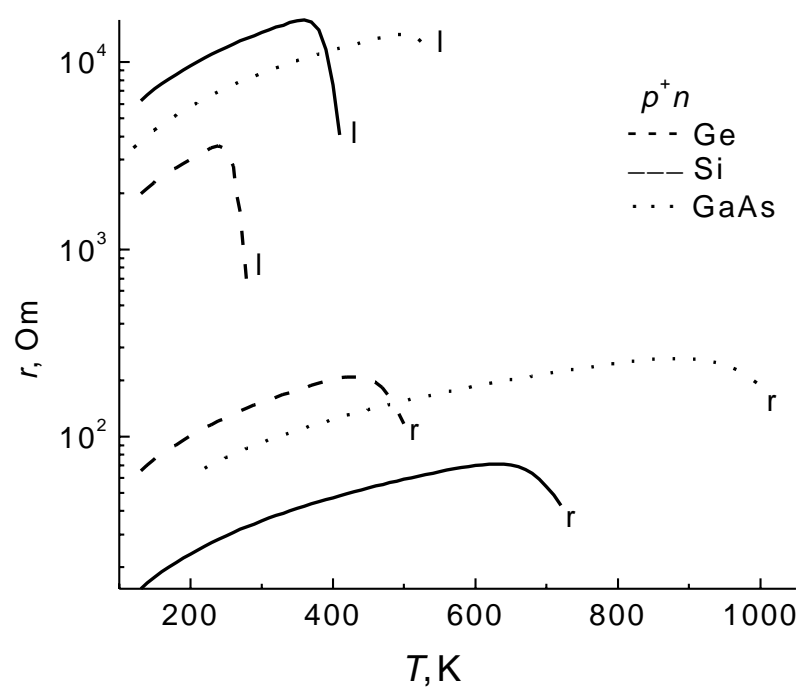

b

Fig. 9. Temperature dependencies of dynamic resistance of Ge, Si, GaAs DTS with abrupt asymmetric $n^{+} p$ (a) и $p^{+} n$ (b) junctions. Legends in the figure: $\mathrm{r}$ - calculation using the parameter set providing the maximum length of TMC; 1 - calculation using the parameter set providing the maximum sensitivity of DTS: 1 - our data for the silicon temperature sensor $\left(I=10 \mu \mathrm{A}, N_{A}==2 \times 10^{17} \mathrm{~cm}^{-3}\right)$.

\section{Conclusion}

Using the diffusion transport model through an asymmetric pn-junction the equations were obtained, for the first time, connecting the length of TMC and sensitivity of the diode temperature sensor with the operation current, semiconductor parameters (minority carrier lifetime and mobility, doping impurity concentration in the base) and parameters of the diode structure ( $p n$-junction area, its depth in respect to the surface contacting with the package, base length). These relations were obtained using a set of fundamental criteria, some of which were formulated for the first time.

The self-consistent consideration of the obtained relations permits to determine the full sets of thermodiode parameter providing required or fixed properties of the thermodiode temperature sensor in the range of validity of the chosen model. In particular, it is shown that fabrication of DTS possessing simultaneously the maximum possible temperature extent of TMC and the highest sensitivity is not possible. The change of parameters leading to the increase of sensitivity always results in shortening of the TMC length and vice versa.

It is found that for the thermometric applications the use of short-base thermodiodes is preferable since in this case both sensitivity and the TMC extent to the high-temperature range is independent of the minority carrier lifetime, which is the least technologically controllable diode parameter.

The effect of the pn-junction location in the chip in respect to the surface being in a contact with a package base on the temperature measurement systematic error due to $p n$-junction Joule heating is analysed. It is found that in the conventional technique of the chip mounting ( $p n$-junction is at the maximum distance from the surface contacting with the package base) this error is maximum. Its lowest value is expected if the $p n$-junction is situated at the minimum distance from the package base, and this distance is shorter than the base length.

The intervals of TMC and sensitivity are determined within which the Ge-, Si- and GaAs-based DTS can be realized. From the comparison of calculated and experimental characteristics TMC and temperature dependencies of sensitivity it follows that none of experimentally realized DST could provide the limiting TMC length or the maximum possible sensitivity.

This work was carried out in the frames of the STCU Project No. 477.

\section{References}

1. N. Sclar, D. B. Pollock // Sol. St. Electr., 15, p.473 (1972).

2. S. P. Logvinenko, T. D. Aluf, T. M. Zarochintseva. Thermometrical characteristics of directly biased $\mathrm{Ge}, \mathrm{Si}$ and GaAs diodes in the range of 4.2-300 K// Kriogennaya $i$ vakuumnaya tekhnika, ser. 2, p.63 (1972) (in Russian).

3. L. Jansak, P. Kordos, M. Blahova. Silicon and gallium arsenide for low-temperature thermometry// Inst. Phys. Conf. 1975, Ser. 26, Chap. N 2, p. 65-69.

4. I. Chopra, G. Dharmadurai. Effect of current on the low temperature characteristics of diode sensors // Cryogenics, 20, p.659 (1980).

5. W. Shockley. The theory of $p n$-junction in semiconductors and pn-junction transistors// Bell System Techn. J., 28, p.435 (1949).

6. V. I. Stafeev. Influence of semiconductor bulk resistance on the form of a diode current-voltage characteristics// ZhTF, 28, p.1631 (1958) (in Russian).

7. J. F. Cerofolini, M. L. Polignano. Residual non-idealities in the almost ideal silicon pn-junction// Appl. Phys., A50, p.273 (1990).

8. S.Sze // Fizika poluprovodnikovykh priborov (Physics of Semiconductor Devices). 1.- M.: Mir, (1984) (Russian translation).

9. S. M. Sze, G. Gibbons. Avalanche breakdown voltage of abrupt and linearly grated pn-junction in $\mathrm{Ge}, \mathrm{Si}, \mathrm{GaAs}$ and $\mathrm{GaP} / / \mathrm{Appl}$. Phys. Lett. , 8, p.111 (1966). 


\section{N. R. Kulish et al.: Self-consistent method for optimization of parameters ...}

10. K. V. Shalimova // Fizika poluprovodnikov (Physics of Semiconductors).- M: Energoatomizdat, (1985) (in Russian).

11. Tables of Physical Constants. Reference book. Ed. by I.K.Kikoin.M.: Atomizdat. (1976).

12. I.V.Fogelson // Tranzistornye termodatchiki (Transistor thermosensors).- M.: Sovetskoe Radio. (1972) (in Russian).

13. Temperature measurement and control, Part 1 of 2 (1995).

14. M. J. Buckingham Noise in electronic devices and systems. Ellis Horwood Ltd. New York, (1983).

15. A. I. Kurnosov, V. V. Yudin // Tekhnologiva proizvodstva poluprovodnikovykh priborov (Technology of manufacture of semiconductor devices). M.: Vysshaya Shkola, (1974) (in Russian).

16. M.Shur // Sovremennye pribory na osnove arsenida galliya (Modern GaAs-based devices). M.: Mir, (1991) (Russian translation).

17. Ye. M. Voronkova, B. N. Grechushnikov, G. I. Distler, I. P. Petrov // Opticheskiye materialy dlia infrakrasnoy tekhniki (Optical materials for infrared technology). M.: Nauka, (1965) (in Russian).

18. Arsenid galliya Poluchenie, svoistva i primenenie (Gallium Arsenide. Fabrication, properties and application) / Ed. by F. P. Kesamanda and D. N. Nasledov / M.: Nauka, (1973) (Russian translation).
19. J. Vanhellemont, E. Simoen, C. Claeys. Extraction of the minority carrier recombination lifetime from forward diode characteristics// Appl. Phys. Lett., 66, p.2894 (1995).

20. A. P. De Fonzo. PIcosecond photoconductivity in germanium film// Appl. Phys. Lett., 39, p.480 (1981).

21. R. B. Phammond, N. G. Paulter, R. S. Wagner. Observed circuit limits to time resolution in correlation measurements with $\mathrm{Si}$-onsapphire, GaAs and InP picosecond photoconductors// Appl. Phys. Lett., 45, p.289 (1984).

22. Von V. Bruckner, F. Kerstan // Exp. Techn. Phys., 32, p.139 (1984).

23. J. Koutny, J. Kulak, J. Mikusek // Tekhnologiya seriynogo proizvodstva tranzistorov i poluprovodnikovykh diodov (Technology of manufacture of transistors and semiconductor diodes). M.: Energiya, (1968) (Russian translation).

24. M. M. Sobolev, V. G. Nikitin. High-temperature diode based on epitaxial GaP layers// Pis'ma v ZhTF, 24, p.1 (1998) (in Russian). 